\title{
Multiscale Belief Propagation on Concrete CT Image Fast Segmentation
}

\author{
Zhao Liang ${ }^{1}$, Lu Jun ${ }^{2}$, Xu Sheng-Jun ${ }^{1}$, Chen Deng-feng ${ }^{1}$, Li chang-hua ${ }^{1}$ and \\ Dang Fa-ning ${ }^{3}$ \\ ${ }^{1}$ Dept of Info and Automation, Xi' an Univ. of Arch. \&Tech., Xi'an 710055 , China \\ ${ }^{2}$ School of Automation and Information Engineering, Xi' an University of Technology, \\ $X i$ 'an 710048, China \\ ${ }^{3}$ Institute of Geotechnical Engineering, \\ $X i^{\prime}$ an University of Technology, Xi'an 710048, China
}

tanatee@163.com

\begin{abstract}
An image segmentation fast method based on multi-scale belief propagation is proposed to solve the concrete CT image segmentations problem. Firstly, according to the feature of belief propagation algorithm, a self-characteristic multiscale belief propagation(MBP) is proposed; Then, according to compute complexity problem in process of belief messages propagation, a method to reduce quantity of algorithm compute is proposed; Finally, using standard images to validate nicety and speediness on our method, and applying on concrete CT image segmentation. The experiment results show that the proposed method can improve efficiency and precision of the image segmentation, and afford an important assisting method on concrete meso-structure CT image study of architecture projection. The method has important projection applying meaning.
\end{abstract}

Keywords: concrete; CT image; image segmentation; multi-scale; belief propagation

\section{Introduction}

Concrete is a kind of special natural defect material, which is consisted of graded aggregate, cement, mortar and holes. The concrete inner construction is complicated, and contains multi-scale, unique physical and mechanical properties. It widely used in the construction industry uneven material.

In recent years, the improvement of test conditions in all aspects, researching of concrete CT image become the focus, and has made some achievements [1, 2, 3]. However, CT images gotten in this way could hardly reflect the complete structure of concrete, for most of which limited to changes in the number of CT images and visual observation of the image, and little is related to the theory.

Image segmentation is always an important issue [4], for improving morphological parameters of concrete cracks to the quantitative description and a deeper exploration of the evolution. The advantage of SBP (Standard Belief Propagation) is convexity of the objective function which is not being limited and lower segmentation error rate; the disadvantage of it is the need for large amounts of information in transmission between the nodes and high computational complexity. When the number of iterations is high, there's a longer execution time. All these above make SBP to be used to solve image problem recent years. 
An image segmentation fast method based on multiscale belief propagation is proposed to solve the concrete CT image segmentations problem in this paper, and improve efficiency and precision of the image segmentation. Compared to ICM, Metropolis, Gibbs sampling [5] and SBP, the new method improves efficiency and precision of the image segmentation efficiently, and be able to reflects the complete internal structure and defects of concrete. It reflects the microstructure of concrete for the full segmentation. Afford an important assisting method on concrete meso-structure CT image study of architecture projection. The method has important projection applying meaning.

\section{Image Model}

MRF is being widely used in edge extraction, segmentation and texture analysis, etc. For a two-dimensional image $S=\left\{s_{1}, s_{2} \cdots, s_{i}, \cdots s_{M}\right\}$ could show the segmentation results of CT images, $\omega_{i}$ stands for the corresponding labels of pixel $i$, and $\omega_{i} \in\{1,2,3, \ldots, L\}, L$ stands for total number classes of the observed image. Since each pixel is corresponding a label, so observed image is corresponding labeling field. Observed image segmentation equals to the label field division. Label problem of observation image can describe by MRF (Markov Random Field) [6].

According to HAMMERSLEY CLIFFORD theorem, Markov Random Fields has homology characteristic with Gibbs Random Fields. So we can confirm Gibbs Random Fields to solve observation image label problem [7]. The Gibbs Random Fields is :

$$
P(\Omega=\omega)=\frac{1}{Z} \exp \left(-\frac{E(\omega)}{T}\right)
$$

$Z$ is constant, $E(\omega)$ is an energy function.

\section{SBP (Standard Belief Propagation) Image Segmentation Algorithm}

For image segmentation, based on Bayes theorem, the image segmentation transform to Maximum a posteriori probability estimation. Here we proposed a belief propagation image method on image segmentation.

For a MFR image model, energy function $E(\omega)$ can denote:

$$
E(\omega)=-\sum_{(i j)} \ln \psi_{i j}\left(\omega_{i}, \omega_{j}\right)-\sum_{i} \ln \phi\left(\omega_{i}, s_{i}\right)
$$

$\phi_{i}\left(\omega_{i}, s_{i}\right)$ is conditional probability of label $\omega_{i}, \psi_{i j}$ apriori probability function of neighborhood labels. We then take overall joint probability of image $S$ and label field $\Omega$ to be

$$
P(\{s\},\{\omega\})=\frac{1}{Z} \prod_{(i j)} \psi_{i j}\left(\omega_{i}, \omega_{j}\right) \prod_{i} \phi_{i}\left(\omega_{i}, s_{i}\right)
$$

$\omega_{i}, \quad \omega_{j}$ is neighborhood label, $s_{i}$ is pixel which is corresponding label $\omega_{i}$.

Suppose $m_{j i}\left(\omega_{i}\right)$ as a "message" from label $j$ to label $i$. For a label node set $\Omega=\left\{\omega_{1}, \omega_{2}, \omega_{3}, \omega_{4}\right\}$, we can count belief of label node $\omega_{1}$ by belief propagation model [8, [9]: $b_{1}\left(\omega_{1}\right)=k \phi_{1}\left(\omega_{1}, s_{1}\right) m_{21}\left(\omega_{1}\right)$. 
Using the message-update rules for $m_{21}\left(\omega_{1}\right)$, we find

$$
b_{1}\left(\omega_{1}\right)=k \phi_{1}\left(\omega_{1}, s_{1}\right) \sum_{\omega_{2}} \psi_{12}\left(\omega_{1}, \omega_{2}\right) \phi_{2}\left(\omega_{2}, s_{2}\right) m_{32}\left(\omega_{2}\right) m_{42}\left(\omega_{2}\right)
$$

Using the message-update rules for $m_{32}\left(x_{2}\right) 、 m_{42}\left(x_{2}\right)$, the belief of label $\omega_{1}$ is

$$
\begin{aligned}
b_{1}\left(\omega_{1}\right)= & k \phi_{1}\left(\omega_{1}, s_{1}\right) \sum_{x_{2}} \psi_{12}\left(\omega_{1}, \omega_{2}\right) \phi_{2}\left(\omega_{2}, s_{2}\right) \sum_{x_{3}} \phi_{3}\left(\omega_{3}, s_{3}\right) \psi_{23}\left(\omega_{2}, \omega_{3}\right) \\
& \sum_{x_{4}} \phi_{4}\left(\omega_{4}, s_{4}\right) \psi_{24}\left(\omega_{2}, \omega_{4}\right)
\end{aligned}
$$

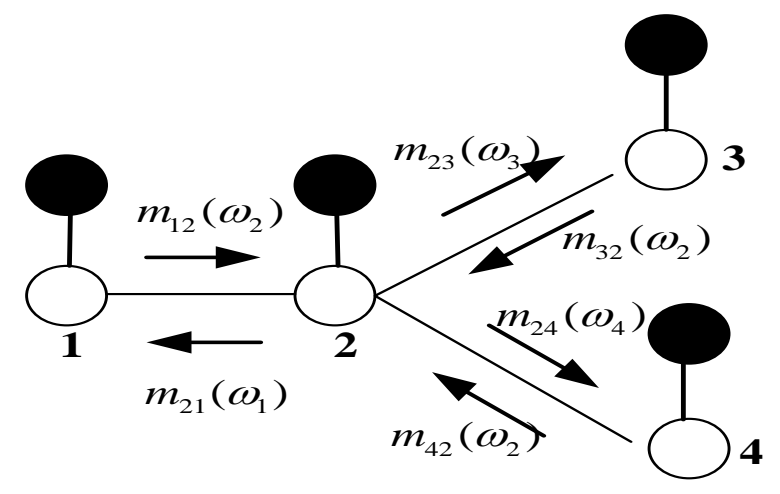

Figure 1. Belief propagation model on 4 label nodes

So from message propagation deduction, we can get message $m_{j i}\left(\omega_{i}\right)$ update rules:

$$
m_{i j}\left(\omega_{j}\right) \leftarrow \sum_{\omega_{i}} \phi_{i}\left(\omega_{i}, s_{i}\right) \psi_{i j}\left(\omega_{i}, \omega_{j}\right) \prod_{k \in N(i) \backslash j} m_{k i}\left(\omega_{i}\right)
$$

Where $k \in N(i) \backslash j$ denotes the nodes neighboring $i$ except node. $j$. The message-update rule is shown diagrammatically in Figure 2.

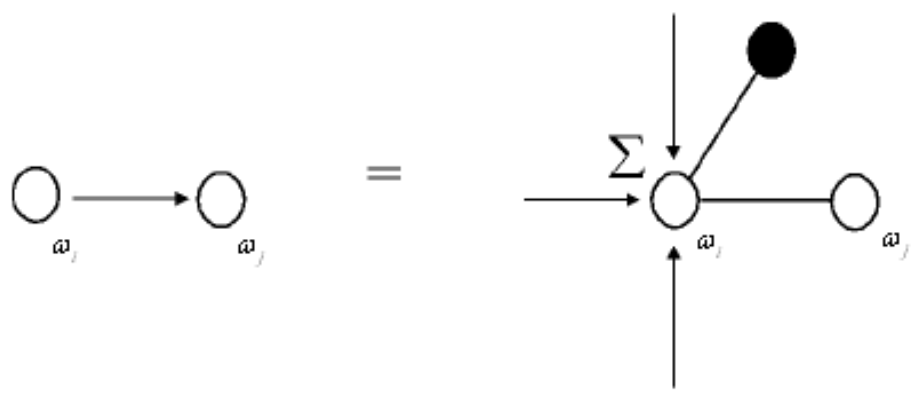

Figure 2. Message-update rules

In the BP algorithm, the belief at a label node $i$ is

$$
b_{i}\left(\omega_{i}\right)=k \phi_{i}\left(\omega_{i}, s_{i}\right) \prod_{j \in N(i)} m_{j i}\left(\omega_{i}\right)
$$


where $k$ is a normalization constant (the beliefs must sum to 1)and $N(i)$ denotes the nodes neighboring $i$ (see Figure 3).

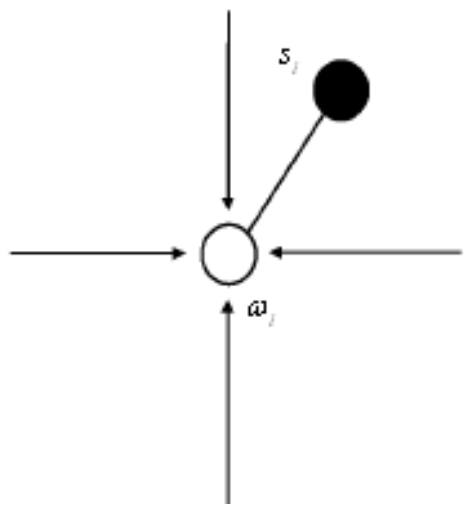

Figure 3. Belief of label node $i$

Here we define the belief $b_{1}\left(\omega_{1}\right)$ is posteriori probability $P\left(\omega_{1}\right)$ of label ${ }^{\omega_{1}}$.From each label field $\Omega=\left\{\omega_{1}, \omega_{2}, \omega_{3}, \ldots, \omega_{N}\right\}$ belief message propagation, by iterative count, we can get posteriori probability and process segmentation of image $S$ [10].

Therefore, using MAP to do image segmentation:

$$
\hat{\omega}=\underset{\omega_{i} \in \Omega}{\arg \max } b_{i}\left(\omega_{i}\right)
$$

\section{Multi-scale Fast Algorithms}

SBP algorithm needs a large number of iterations to achieve convergence information. With the increase of the number of iterations, the algorithm execution time increase. MBP (Multi-scale Belief Propagation) is an accurate way, which could reduce the iterations and improve the classification accuracy. We do not change the neighborhood structure of the case, to reduce iterations of information propagation in the scale of each layer. In other words if we get a certain level of belief scale, you can use the layer belief information to estimate more accurate information on other scale layer of belief $[11,12]$.

The original image $S$ can be regarded as grid $\Gamma$. Each coordinates $(x, y)$ of grid to be defined label $\omega_{x, y} \in \boldsymbol{\Omega}$, and Probability function is:

$$
\begin{aligned}
P(\{\omega\}) & =\frac{1}{Z} \prod_{(x y) \in \Gamma} \phi_{x, y}\left(\omega_{x, y}\right) \prod_{(x y) \in \Gamma \backslash C} \psi_{x, y}\left(\omega_{x, y}, \omega_{x+1, y}\right) \\
& \times \prod_{(x y) \in \Gamma \backslash R} \psi_{x, y}\left(\omega_{x, y}, \omega_{x, y+1}\right)
\end{aligned}
$$

$\phi_{x, y}\left(\omega_{x, y}\right)_{\text {simplify of }} \phi_{x, y}\left(\omega_{x, y}, s_{x, y}\right), \mathrm{C}$ and $\mathrm{R}$, respectively, is the last line of the grid and the last row. 
Assuming the ground $\Gamma^{0}, \Gamma^{1}, \cdots, \Gamma^{l}, \cdots$ is fine to coarse grid map collection, which indicates that the original figure $\Gamma^{0}$ is a measure of the finest layer, $\Gamma^{l}$ is $l$ layer of a scale. Each node (pixel block) of which is constituted by $\alpha \times \alpha$ pixels, $\alpha=2^{l}$. So we can replace the transmission of information of each pixel with pixel blocks.

Message transmission in pyramid-shaped structure not only improves the interactivity of pixel blocks in different shapes and lays, but also fastens the convergence rate, thus substantially reducing the number of iterations required. As shown in Figure 4.
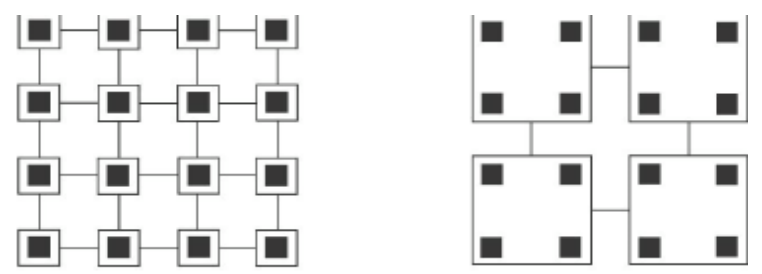

Figure 4 (a). Nodes at scale $l$ Figure 4 (b). Node blocks at scale $l+1$

Figure 4. Structure of two neighboring scales

Belief messages propagation in each scale layer is iterative transfer; node ${ }^{j}$ (pixel block) to the neighborhood node $i$, the message in the first $t$ iterations is expressed as:

$$
m_{j \rightarrow i}^{l, t}\left(\omega_{i}\right)=\sum_{\omega_{j}} \phi_{j}^{l}\left(\omega_{j}\right) \psi_{i j}^{l}\left(\omega_{i}, \omega_{j}\right) \prod_{k \in N(j) \backslash i} m_{k \rightarrow j}^{l, t-1}\left(\omega_{j}\right)
$$

Any node $i$ in the $\Gamma^{l}$ node label $\omega_{i} 1$ is equivalent to the corresponding labels of pixels. $\phi_{j}^{l}\left(\omega_{j}\right)$ is corresponding conditional probability of nodes $\mathrm{j}$ on the $l$ layer,

$$
\phi_{j}^{l}\left(\omega_{j}\right)=\sum_{u=0}^{\alpha-1} \sum_{v=0}^{\alpha-1} \phi_{j_{x} \times \alpha+u, j_{y} \times \alpha+v}^{0}\left(\omega_{j}\right)
$$

$\left(j_{x}, j_{y}\right)_{\text {means that the node }}^{j}$ coordinate of $\Gamma^{l}$ scale layer. $\psi_{i j}^{l}\left(\omega_{i}, \omega_{j}\right)$ Means the adjacent node's probability function:

$$
\psi_{i j}^{l}\left(\omega_{i}, \omega_{j}\right)=\alpha \psi_{i j}\left(\frac{\omega_{i}, \omega_{j}}{\alpha}\right)
$$

$\alpha$, outside of the function, is the number of pixels of adjacent nodes edge, within the $\psi_{i j}$ function, is center distance of adjacent nodes. As each layer of pixels in the same node with the same label, segmentation parameters $\beta$ exist only between nodes with different.

In the belief that the process of information transmission, the standard BP algorithm needs time to run is $O\left(n k^{2} T\right), n$ is Image pixel, $k$ is the possible corresponding label number of each pixel, $T$ is the number of iterations. We can draw that it's $O\left(k^{2}\right)$ that a computer needs to calculate for information and there're $O(n)$ information to calculate in iteration each time. 
Here a two-way technology which could reduce the amount of information and belief will not affect the results of the image segmentation. First, divide all the MBP nodes into two sets $\mathrm{A}$ and $\mathrm{B}$ in each scale layer, as shown in Figure 5.

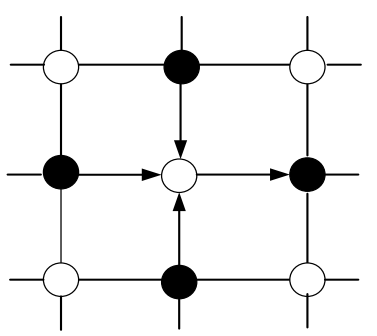

Figure 5(a). Filled-in circles pass messages to empty circles

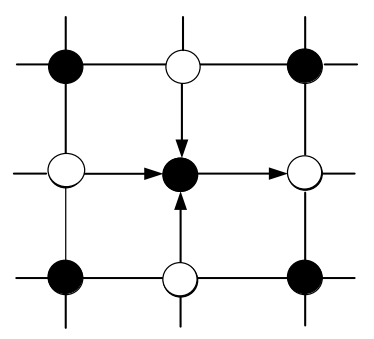

Figure 5(b). Empty circles pass messages to filled-in circles

Figure 5. Messages propagation model

A solid circle represents one of the nodes, the hollow circle represents the node $\mathrm{B}$, in this way, and the ways message transmission are only two: $A \rightarrow B$ (Figure 5 (a))and $B \rightarrow A$ (Figure 5 (b)), in each iteration, you could just choose to update one message, if in the time iterations to update the message A node, then you can calculate the time $t+1$ iterations of the message node $\mathrm{B}$, and iterations in the $t+2$ only to updates node $\mathrm{A}$, cycle this step.

In this information update mode of the two-way technology, belief information $\bar{m}_{i \rightarrow j}^{t}$ is information from node $i$ to node ${ }^{j}$ on a scale layer in the iteration $t$. If $t$ is odd number. We only update the node information in the set $\mathrm{A}$ and node information of set $\mathrm{B}$ remains. If ${ }^{t}$ is even number. We only update the node information in the set $\mathrm{B}$ and node information of set $\mathrm{A}$ remains. Based on this, each time we only need to calculate half of the belief information, and because updates information are not related to each other, so the new updated information stored in the old information memory space.

The message's formula expression is:

$$
\bar{m}_{i \rightarrow j}^{t}= \begin{cases}m_{i \rightarrow j}^{t} & \text { if } i \in A(i \in B) \\ m_{i \rightarrow j}^{t-1} & \text { otherwise }\end{cases}
$$

When MBP is convergent, this interactive information updates and converges to a fixed point $m_{i \rightarrow j}^{t-1}=m_{i \rightarrow j}^{t}$.

Therefore, this method can decrease the computational complexity from $O\left(n k^{2} T\right)$ to $O\left(n / 2 k^{2} T\right)$, and the space complexity of storing information has also been reduced.

\section{Image Segmentation and Experimental Analysis}

Standard image and concrete CT images were used for validation and comparison. The experiment was done by using an inter dual-core $1.66 \mathrm{GH}$, memory $2 \mathrm{~GB}$ computer. 


\subsection{Standard Image Segmentation experiment}

In the experiment, we used ICM algorithm, Gibbs sampling algorithm, Metropolis sampling algorithm, SBP algorithm and MBP for segmenting the sample image and making a comparison. Experiment sample image selecting synthetic images and natural images. Synthetic triangle images $(128 \times 128, \mathrm{SNR}=-5 \mathrm{~dB})$ added with noise were divided into four categories. Natural beach $(200 \times 300)$ grayscale images were divided into five categories. In the experiment, for ICM algorithm, the temperature parameters is a constant, for other Gibbs sampling algorithms, Metropolis sampling algorithms, set $T^{(t+1)} \leftarrow 0.95 T^{(t)}$, and make the initial value $T^{(0)} \leftarrow 8$.MBP algorithm for a scale model was divided into five layers. Gaussian mixture parameter was estimated by EM algorithm [13], segmentation parameters $\beta$ were given by the experiment. Figure 6 (a) for different ways of segmentation of add noise image $\beta=2$. Figure 6(b) for different ways of segmentation of natural image $\beta=3$. Table 1 gives the comparison between this algorithm and several other segmentation methods about recognition rate and time performance.

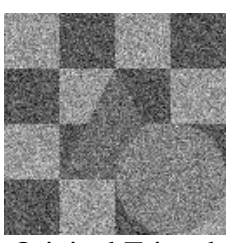

Original Triangle

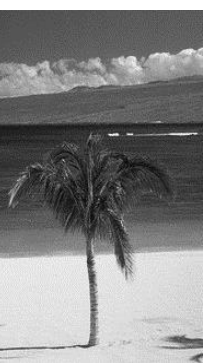

Original Bench

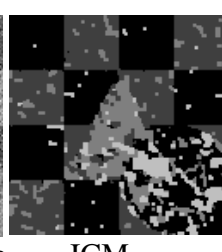

ICM

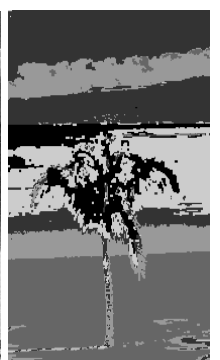

ICM

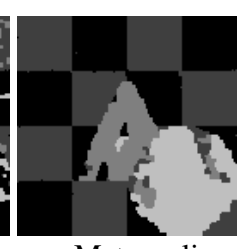

Metropolis

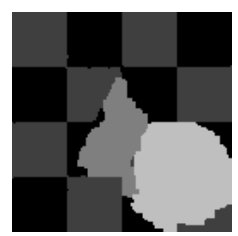

Gibbs sampler

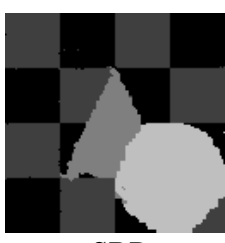

SBP

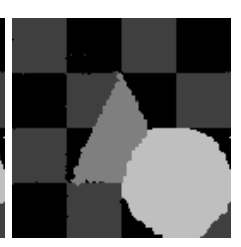

MBP

Figure 6. (a) triangle segment

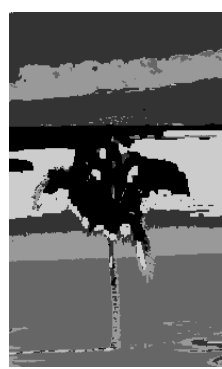

Metropolis

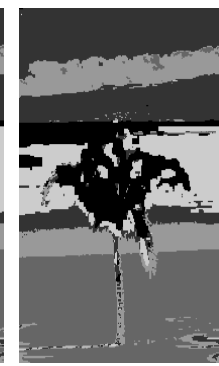

Gibbs sampler

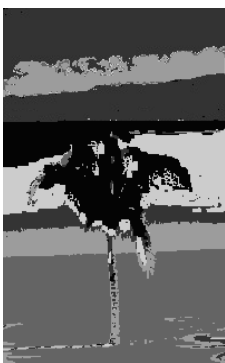

SBP

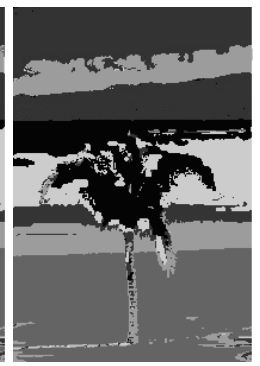

MBP

Figure 6. (b) Bench segment

Figure 6. Result of standard image segment

As we can be seen from Table 1 that MBP algorithm and the SBP algorithm are similar in recognition rate, but since the SBP algorithm has no scaling transformation and MBP algorithm adopts a two-way graph technique that improved its efficiency. So SBP algorithm need more time to get recognize in Table 1 (the number of Triangle iteration need above 20, the number of Bench iteration need above 150 times). Compared with ICM, Gibbs sampling and Metropolis, ICM iteration shows its least times, fast convergence, but largest error. The time that Gibbs sampling involved is close to SBP algorithm. Metropolis is faster in convergence than SBP and Gibbs sampling, but MBP algorithm shows a better segmentation effect, so MBP algorithm is superior above three algorithms in segmentation effect and segmentation efficiency. 
Table 1. Comparison recognition rates and time of different methods

\begin{tabular}{ccccc}
\hline \hline \multirow{2}{*}{ methods } & \multicolumn{2}{c}{ triangle } & \multicolumn{2}{c}{ bench } \\
\cline { 2 - 5 } & Total time/s & Recognize\% & Total time/s & Recognize\% \\
\hline ICM & 0.15 & 87.6 & 3.66 & 84.6 \\
Metropolis & 2.10 & 92.8 & 11.52 & 88.93 \\
Gibbs sampler & 2.59 & 94.5 & 32.50 & 89.61 \\
SBP & 2.78 & 98.1 & 40.87 & 92.56 \\
MBP & 1.47 & 99.3 & 10.66 & 94.78 \\
\hline \hline
\end{tabular}

\subsection{Concrete CT image segmentation}

Concrete CT image adopted Marconi M 8000 spiral CT scanner of Xi'an Central Hospital Imaging Center to scanning, Scan Thickness $2.5 \mathrm{~mm}$. Use Instron1342 machine to do the compression test. Loading rate is $1.2 \times 10-3 \mathrm{kN} / \mathrm{s}$.

CT images of concrete structure is complex, especially in the internal structure of the press will occur after the corresponding changes. The main observation in the concrete is aggregate, mortar, holes and cracks. Holes and cracks which are most concerned about the concrete structure. The number of cracks and crack distribution can be used to determine whether the damaged concrete. Because of the aggregate density is heavy, the bright regional in the images are the aggregate area. We can see aggregate shape and the spatial distribution of aggregate section position, but aggregate regional boundary is difficult to distinguish. The brightness of Mortar regional is slightly lower than aggregate regional. However, some tiny area with higher brightness distribute in Mortar regional, which is small gravel with heavier density in mortar. The lowest brightness area in Section is hole and crack area, the hole has been generated during the preparation process of specimens, its distribution is extremely indiscipline.

Due to limited space, take a section of four stress levels of concrete CT image $(1024 \times 1024)$ to segment. Segmentation results shown in Figure 7, in various methods: $\beta=5$. Segmentation results can show that as the pressure increases, the concrete structure will change, the crack appears. Compared with SBP, this method could get better segmentation of concrete aggregate, mortar, holes and cracks, in particular, when the division of the regional aggregate and mortar is not clear enough. It has an important role in supporting analysis of changes of concrete structures with press. The convergence time of image segmentation of SBP is 74.12s, while MBP is $32.15 \mathrm{~s}$.

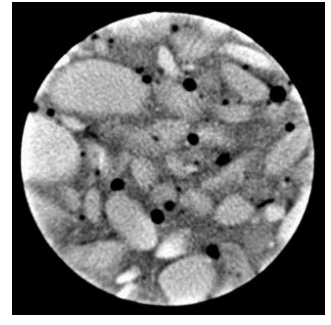

press $\sigma=\mathrm{OMPa}$

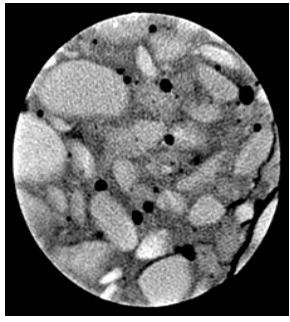

press $\sigma=17.69 \mathrm{MPa}$

Original concrete $\mathrm{CT}$ image

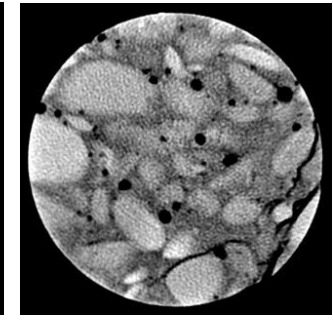

press $\sigma=21.92 \mathrm{MPa}$

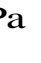

press $\sigma=28.72 \mathrm{MPa}$

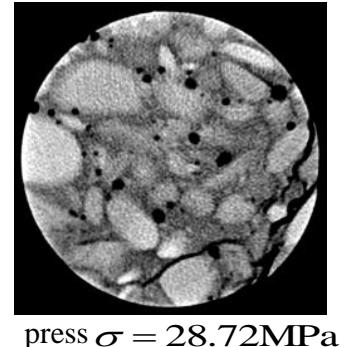

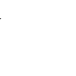



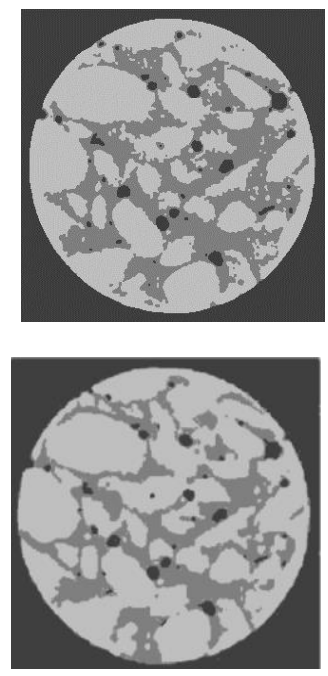
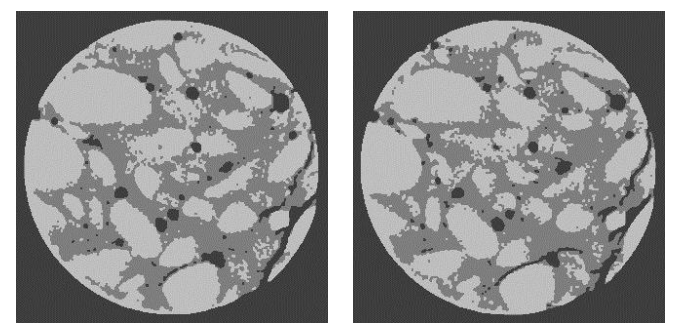

SBP segment
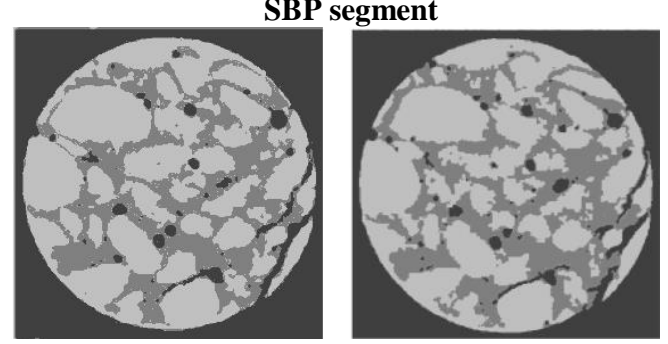

MBP segment
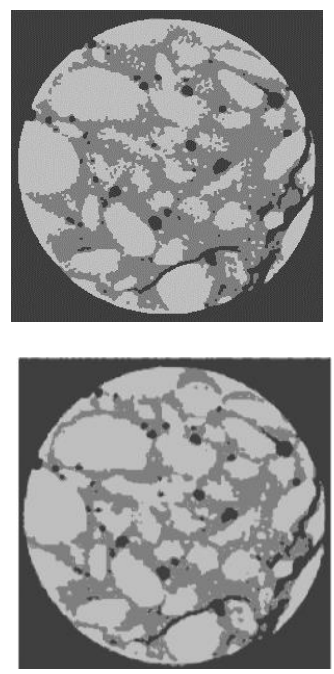

Figure 7. Result of concrete CT image segment

\section{Conclusions}

An image segmentation fast method based on multistage belief propagation is proposed to solve the concrete CT image segmentations problem. Through comparing with ICM 、 Metropolis、Gibbs and SBP segmentation method, what can be inferred from the result is this new method can lead to a faster and more accurate image segmentation. For the segmentation of the concrete structure with press, the method can segment the concrete structural components and distribution of fine cracks, and make a significant improvement in image segmentation. The new method affords an important assisting method on concrete meso-structure CT image study of architecture projection, and has an important applied significance in determining whether the concrete stress is damaged in construction.

\section{Acknowledgements}

This work was financially supported by National Nature Science Foundation of China (Grant No.51209167), and was supported by Nature Science Foundation of Shaanxi Province (Grant No. 2013JQ7024)

\section{References}

[1] D. Fa-ning, L. Yan-wen, D. Wei-hua et al., "Quantative analysis of concrete CT image based on damagefracture evolution theory", Chinese Journal of Rock Mechanics and Engineering, vol. 22, no. 8, (2007), pp. 142-147.

[2] T. Wei, D. Fa-ning and L. Xin-yu, "CT image analysis of meso fracture process of concrete", Engineering Journal of Wuhan University, vol. 41, no. 2, (2008).

[3] L. Xin-yu, D. Fa-ning and T. Wei, "CT testing on fracture process of concrete under uniaxial compression", Journal of China Coal Society, vol. 35, (2010).

[4] W. Huai-bin, W. Xian-bin, L. V. Yang-li, et al., "Segmentation for SAR image based on self-organizing mixture network", Journal of Optoelectronics Laser, vol. 18, no. 4, (2007).

[5] L. Zhao, C. -h. Li, F. -n. Dang, et al., "Architecture concrete mesostructure analysis based on CT image fracture process", Journal of Xi an University of Architecture \& Technology(Natural Science Edition), vol. 42 , no. 5, (2010).

[6] M. Tanak and M. Okutomi, "Locally adaptive leaning for translation-variant MRF image priors", IEEE Conference on Computer Vision and Pattern Recognition, (2008) June 23-28; CA, USA. 
[7] Z. Liang, L. Chang-hua, D. Fa-ning, et al., "Concrete CT Image MMD Segmentation Method Based on Markov Random Field”, Acta Photonica Sinica, vol. 39, no. 9, (2010).

[8] B. Poteztz and T. S. Lee, "Efficient belief propagation for higher order cliques using linear constraint nodes", Computer Vision and Image Understanding, vol. 112, no. 1, (2008).

[9] S. Y. Jonathan, T. F. William and W. Yair, "Generalized Belief Propagation", Advances in Neural Information Processing Systems (NIPS), vol. 13, no. 7, (2000).

[10] P. F. Felzenszwalb and D. P. Huttenlocher, "Efficient Belief Propagation for Early Vision", IEEE Computer Society Conference on Computer Vision and Pattern Recognition (CVPR'04), (2004) June 27 - July 2; Washington DC, USA.

[11] S. Y. Jonathan, T. F. William and W. Yair, "Constructing free-energy approximations and generalized belief propagation algorithms", IEEE Transactions on Information Theory, vol. 51, no. 7, (2005).

[12] X. Ren and J. Malik, "Learning a classification model for segmentation", Ninth IEEE International Conference on Computer Vision Proceedings, (2003) October 13-16, Nice, France.

[13] F. Chen, K. Tanaka and T. Horiguchi, "Image Segmentation Based on Bethe Approximation for Gaussian Mixture Model”, Interdisciplinary Information Sciences, vol. 11, no. 1, (2005).

[14] K. Saini, M. L. Dewal and M. Rohit, "Ultrasound Imaging and Image Segmentation in the area of Ultrasound: A Review", International Journal of Advanced Science and Technology, vol. 24, (2010) November, pp. 41-60.

[15] C. H. Bindu and K. S. Prasad, "An Efficient Medical Image Segmentation Using Conventional OTSU Method", International Journal of Advanced Science and Technology, vol. 38, (2012) January, pp. 67-74.

[16] W. A. Fourati and M. S. Bouhlel, "Trabecular Bone Image Segmentation Using Iterative Watershed and Multi Resolution Analysis”, International Journal of Bio-Science and Bio-Technology, vol. 3, no. 2, (2011) June, pp. 71-82.

[17] W. Watthayu, "Loopy Belief Propagation: Bayesian Networks for Multi-Criteria Decision Making (MCDM)", International Journal of Hybrid Information Technology, vol. 2, no. 2, (2009) April, pp. 141-152. 